\title{
Pacific
}

Journal of

Mathematics

\section{OSCILLATORY THEOREM AND PENDENT LIQUID DROPS}

KIMIAKI NARUKAWA AND TAKASHI SUZUKI

Volume $176 \quad$ No. 2

December 1996 


\section{OSCILLATORY THEOREM AND PENDENT LIQUID DROPS}

\section{KIMIAKI NARUKAWA AND TAKASHI SUZUKI}

In this paper the bifurcation diagram for the equation of a pendent liquid drop is devoted. The bendings of the branch of solutions bifurcated from the trivial solution occur infinitely many times.

\section{Introduction.}

In this article we discuss the bifurcation problem for a model equation which describes capillary surfaces. The membrane in the equilibrium state which is framed horizontally and filled up with fluid in the gravity field yields to the equation of mean curvature type

$$
-\operatorname{div}\left(\frac{\nabla u}{\sqrt{1+|\nabla u|^{2}}}\right)=\lambda u \quad \text { in } \Omega
$$

with the boundary condition

$$
u=0 \quad \text { on } \partial \Omega
$$

and the constraint

$$
u<0 \quad \text { in } \Omega,
$$

where $\Omega$ is a bounded domain in $R^{2}, \lambda$ a positive parameter determined by the physical constants.

Here $z=u(x, y),(x, y) \in \Omega$, represents the shape of the membrane spanned by $\partial \Omega$. Actually, the left hand side in Equation (1.1) defines the mean curvature of a graph $(x, y, u(x, y)),(x, y) \in \Omega$ at each point.

Noting that $u \equiv 0$ is the trivial solution for any $\lambda>0$, we see that nontrivial solutions of (1.1), (1.2), (1.3) bifurcate from $u \equiv 0$ at the first eigenvalue $\lambda=\lambda_{0}$ of the linearized equation

$$
-\Delta u-\lambda u=0 \quad \text { in } \Omega
$$

with

$$
u=0 \quad \text { on } \partial \Omega,
$$


by the usual method. The branch of solutions is subcritical, i.e., there exists a solution for any $\lambda$ with $\lambda_{0}-\delta<\lambda<\lambda_{0}$ for small $\delta>0$. Moreover, we can show that there are no solutions of (1.1), (1.2), (1.3) for $\lambda \geq \lambda_{0}$ (see the part at the beginning of Section 2). However, we do not know how the branch of solutions behaves globally by this method of bifurcation theory. When the global behavior of the branch of solutions is regarded, we would like to consider an equation

$$
H[X](p)=\lambda X^{3}(p) \quad \text { in } B_{1} \equiv\left\{p \in R^{2}|| p \mid<1\right\}
$$

instead of (1.1). Here $X(p)=\left(X^{1}(p), X^{2}(p), X^{3}(p)\right), p \in B_{1}$, represents the deformed surface in parametrized form and $H[X](p)$ denotes the mean curvature of the surface at each point $X(p)$. In fact, $(1.1)$ is a reduced form of (1.6) when the surface $\left\{X(p) \mid p \in B_{1}\right\}$ is represented by a graph: $X_{3}=$ $u\left(X_{1}, X_{2}\right)$.

In this paper we consider the case when the frame is given by a horizontal unit circle, namely, (1.6) with

$$
\left(X^{1}(p), X^{2}(p)\right) \in \partial B_{1} \quad \text { and } \quad X^{3}(p)=0 \quad \text { on } \partial B_{1}
$$

and

$$
X^{3}(p)<0 \quad \text { in } B_{1} .
$$

Wente [15] has shown that the surfaces given by Equations (1.6), (1.7), (1.8) are the ones of revolution with $x_{3}$-axis as the axis of symmetry. Then the generating curve $u$ of each surface is given by smooth continuations of arcs determined by solutions of the ordinary differential equation

$$
\left(\frac{r u_{r}}{\sqrt{1+u_{r}^{2}}}\right)_{r}=-\lambda \operatorname{sgn}\left(u_{r}\right) r u
$$

in terms of radial distance $r$ from the axis of symmetry with the boundary conditions

$$
u_{r}(0)=0, \quad u(1)=0
$$

and the constraint

$$
u(r)<0 \quad \text { on }[0,1)
$$


where subindex $r$ indicates the derivative with respect to $r$. Equation (1.9) can be rewritten in terms of the arc length $s$ along the trajectory as

$$
\left\{\begin{array}{l}
\frac{d r}{d s}=\cos \psi \\
\frac{d u}{d s}=\sin \psi \\
\frac{d \psi}{d s}=-\lambda u-\frac{\sin \psi}{r}
\end{array}\right.
$$

where $\psi$ is the angle of inclination of the solution curve with regard to the $r$ axis. In this case we can avoid the argument of the continuation of solutions. Physical backgrounds and many results concerning Equations (1.9) or (1.12) are stated in detail in the monograph [7] by Finn. And further various properties on solutions of the type of this equation have been investigated precisely by many authors, i.e., ground state solutions $[\mathbf{2}],[\mathbf{1 0}],[\mathbf{1 1}],[\mathbf{1 4}]$, a singular solution [4], [5], and vertical points of solutions [1], [3], etc.

In the paper [13], Narukawa and Suzuki have shown the existence of the lower bound $\underline{\lambda}>0$ for which solutions of (1.9), (1.10), (1.11) exist, and Finn [9] has given a new proof of this fact by using the method of Green's identities. Finn [9] further shows that the branch of solutions $\left(\lambda, u_{\lambda}\right)$ blows up at $\lambda=\lambda_{*}$ with $\lambda_{*}$ given by $R_{\infty}^{2} \sim 1.32^{2}$, where $R_{\infty}$ is the first zero of singular solution of (1.9) for $\lambda=1$. Here computational calculation suggests that the branch bends infinitely many times at $\lambda=\lambda_{*}$, but this result of oscillation has not yet proved mathematically. It is our aim to give a partial answer for this.

\section{Main results.}

Prior to starting the main theorems, we remark that there are no solutions of (1.1), (1.2), (1.3) for $\lambda \geq \lambda_{0}$, where $\lambda_{0}$ is the first eigenvalue of $-\Delta$ with the Dirichlet boundary condition. In fact, let $u$ be a solution of (1.1), (1.2), (1.3) and $\lambda(u)$ and $\varphi(x)$ be the first eigenvalue and eigenfunction corresponding to this $\lambda(u)$ respectively, of the linear equation

$$
-\operatorname{div}\left(\frac{\nabla \varphi}{\sqrt{1+|\nabla u|^{2}}}\right)=\lambda \varphi \quad \text { in } \Omega
$$

with

$$
\varphi=0 \quad \text { on } \partial \Omega
$$


Multiplying Equations (1.1) and (2.1) by $\varphi$ and $u$ respectively and integrating over $\Omega$, we have

$$
\int_{\Omega} \frac{\nabla u \nabla \varphi}{\sqrt{1+|\nabla u|^{2}}} d x=\lambda \int_{\Omega} u \varphi d x
$$

and

$$
\int_{\Omega} \frac{\nabla u \nabla \varphi}{\sqrt{1+|\nabla u|^{2}}} d x=\lambda(u) \int_{\Omega} u \varphi d x
$$

Since $\varphi(x)>0$ in $\Omega$ from the Krein-Rutman theorem and $u(x)<0$ in $\Omega$ from (1.3), we have

$$
\int_{\Omega} u \varphi d x<0 .
$$

And hence $\lambda=\lambda(u)$. On the other hand,

$$
\begin{aligned}
\lambda(u) & =\inf \left\{\left.\int_{\Omega} \frac{|\nabla \varphi|^{2}}{\sqrt{1+|\nabla u|^{2}}} d x\left|\varphi \in H_{0}^{1}(\Omega), \int_{\Omega}\right| \varphi\right|^{2} d x=1\right\} \\
& <\inf \left\{\left.\int_{\Omega}|\nabla \varphi|^{2} d x\left|\varphi \in H_{0}^{1}(\Omega), \int_{\Omega}\right| \varphi\right|^{2} d x=1\right\} \\
& =\lambda_{0},
\end{aligned}
$$

where $\lambda_{0}$ is the first eigenvalue of $-\Delta$ with the Dirichlet boundary condition. This implies that there are no solutions of (1.1), (1.2), (1.3) for $\lambda \geq \lambda_{0}$.

Therefore the branch of solutions which bifurcates from the trivial solution at $\lambda=\lambda_{0}$ does not reach into the region $\lambda \geq \lambda_{0}$. Actually, allowing multivalued functions as solutions, we have the following two theorems.

Let $X$ be the set of smooth curves $C$ connecting $(1,0)$ and $\left(0, u_{0}\right), u_{0}<0$, in the half plane $\left\{(r, u) \in R^{2} \mid r \geq 0\right\}$. It is equipped with the metric defined by

$$
d_{X}\left(C_{1}, C_{2}\right)=\max \left\{\max _{p=(r, u) \in C_{1}} \operatorname{dist}\left(p, C_{2}\right), \max _{p^{\prime}=\left(r^{\prime}, u^{\prime}\right) \in C_{2}} \operatorname{dist}\left(p^{\prime}, C_{1}\right)\right\}
$$

for $C_{1}, C_{2} \in X$. We regard the arc $C_{\lambda}$ whose revolution with $u$-axis is a surface given by Equations (1.6), (1.7), (1.8) as an element in $X$. Namely, $C_{\lambda} \in X$ represents the smooth continuation of arcs given by solutions (1.9) with (1.10), (1.11) or a curve $(r(s), u(s))$ given by $(1.12)$ with $(r(0), \psi(0))=$ $(0,0),\left(r\left(s_{1}\right), u\left(s_{1}\right)\right)=(1,0)$ for some $s_{1}>0$ and $u(s)<0$ on $0 \leq s<s_{1}$ for given $\lambda>0$.

Theorem 2.1. The set of solutions $S=\left(\lambda, C_{\lambda}\right)$ forms a 1-dimensional manifold in $R \times X$, homeomorphic to $R$, starting from $\left(\lambda_{0}, 0\right)$ and approaching 
$\lambda=\lambda_{*}$ as the minimum of $u$-component of $C_{\lambda}$ goes up to $-\infty$. Here, $\lambda_{*}=$ $R_{\infty}^{2} \sim 1.32^{2}$ with the first zero $R_{\infty}$ of the singular solution $U(r)$ of $(1.9)$ for $\lambda=1$ which is obtained in [8].

Theorem 2.2. The bendings with respect to $\lambda$ on $S$ in $R \times X$ occur infinitely many times in any neighborhood of $\lambda=\lambda_{*}$.

Theorem 2.1 is already known, more or less. In fact, by the similar transformation $\ell=\sqrt{\lambda} r, v=\sqrt{\lambda} u$, Equation (1.9) is normalized as $\lambda=1$. Further, taking $v$ as an independent variable and $\ell$ as a function of $v,(1.9)$ turns to

$$
\left(\frac{\ell \ell_{v}}{\sqrt{1+\ell_{v}^{2}}}\right)_{v}=\ell v+\sqrt{1+\ell_{v}^{2}} .
$$

Taking account of the boundary condition (1.10) and the constraint (1.11), we see that a solution curve $C_{\lambda}$ corresponds to the solution of $(2.5)$ on $\left(v_{0}, 0\right]$ with some $v_{0}<0$ which satisfies the boundary condition

$$
\ell\left(v_{0}\right)=0, \quad \lim _{v \downarrow v_{0}} \ell^{\prime}(v)=\infty
$$

and

$$
\ell(0)=\sqrt{\lambda}
$$

We also notice that the local existence and the uniqueness of solutions of (2.5) with the initial condition (2.6) have been proved by Wente [16] and Concus and Finn [6] (see also Sections 4.2 and 4.3 in [7]). Further a local solution is continued globally on $\left[v_{0}, 0\right]$ as a solution of (2.5) in a single valued function of $v$, which is denoted by $\ell\left(v, v_{0}\right)$, and the mapping from $v_{0}$ to $\ell\left(0, v_{0}\right)$ is smooth. Thus, for each $v_{0}<0$, the solution $\ell\left(v, v_{0}\right)$ corresponds to a curve $C_{\lambda}, \lambda=\ell\left(0, v_{0}\right)^{2}$, by the relation

$$
C_{\lambda}:\left\{\begin{array}{l}
r=\frac{1}{\sqrt{\lambda}} \ell\left(\frac{v}{\sqrt{\lambda}}, \frac{v_{0}}{\sqrt{\lambda}}\right) \\
u=\frac{v}{\sqrt{\lambda}}
\end{array}\right.
$$

Namely, the branch of solutions $S$ is a family parametrized by $v_{0}<0$ and the minimum of $u$-component of $C_{\lambda}$ is $v_{0} / \sqrt{\lambda}$. Hence, from the continuity of solutions on initial data, the curve $\widetilde{S} \equiv\left\{\ell\left(0, v_{0}\right)^{2}, v_{0} / \ell\left(0, v_{0}\right) \mid v_{0}<0\right\}$ in $R^{2}$ defined by the solutions $\ell$ of $(2.5),(2.6)$ indicates the branch of solutions $S$. Finn [7] notes that $\ell\left(0, v_{0}\right)$ converges to the square of the first zero of the Bessel function $J_{0}(r)$, which is the first eigenvalue of $-\Delta$ in $B_{1}$ with the 
Dirichlet boundary condition. This shows that the branch of solutions $S$ parametrized by $v_{0}$ as above is the one which bifurcates at $\lambda=\lambda_{0}$. Hence our problem is to investigate the behavior $\ell\left(0, v_{0}\right)$, that is, how $\ell\left(0, v_{0}\right)$ approaches $R_{\infty}$, as $v_{0}$ tends to $-\infty$.

Therefore, Theorem 2.1 follows from a theorem proved by Finn [8], which is nothing but Conjecture 3 in [7]. This shows that $\ell\left(v, v_{0}\right)$ converges uniformly to a singular solution $\ell_{\infty}(v)$ with all derivatives in any compact set in $(-\infty, 0]$. Here $\ell_{\infty}(v)$ is a positive global solution of $(2.5)$ on $(-\infty, 0]$ satisfying $\ell_{\infty}(v) \rightarrow 0$ as $v \rightarrow-\infty$. Hence, when $v_{0}$ goes to $-\infty, \lambda=\ell\left(0, v_{0}\right)^{2}$ tends to $\lambda_{*}=\ell_{\infty}(0)^{2}=R_{\infty}^{2}$. Thus Theorem 2.1 is proven.

\section{Proof of Theorem 2.2.}

For the solution $\ell\left(v, v_{0}\right)$ of $(2.5)$ with $(2.6)$, let us put

$$
\phi\left(v, v_{0}\right)=\frac{\partial \ell}{\partial v_{0}}\left(v, v_{0}\right), \quad\left(v_{0}<v<0\right) .
$$

Then the branch of solutions $S$ bends at $\lambda=\ell\left(0, \bar{v}_{0}\right)^{2}$ with respect to $\lambda$ if and only if there exists an interval $J=[a, b]$ containing $\bar{v}_{0}$ such that $\phi\left(0, v_{0}\right)$ vanishes on $J$ and changes its sign on a neighborhood of $J$.

Our strategy is to look at the number of zero points of $\phi\left(\cdot, v_{0}\right)$ in the interval $\left(v_{0}, 0\right]$. We shall show that it goes to infinity as $v_{0} \rightarrow-\infty$, since the variational equation for the singular solution is oscillatory. On the other hand, it will be also shown that the zeros of $\phi\left(\cdot, v_{0}\right)$ never appear afresh in any compact subinterval of $\left[v_{0}, 0\right)$. This indicates that $\phi\left(0, v_{0}\right)$ must change its sign when the number of zeros of $\phi\left(\cdot, v_{0}\right)$ changes, so that $\phi\left(0, v_{0}\right)$ changes its sign infinitely many times as $v_{0} \rightarrow-\infty$.

We begin with some lemmas.

Lemma 3.1. For each $v_{0}<0$, the function $\phi\left(v, v_{0}\right)$ satisfies the variational equation

$$
\left[\frac{\ell}{\left(1+\ell_{v}^{2}\right)^{3 / 2}} \phi_{v}\right]_{v}+\left(\frac{\ell_{v}}{\sqrt{1+\ell_{v}^{2}}}\right)_{v} \phi=v \phi
$$

on the interval $\left(v_{0}, 0\right]$, and further,

$$
\lim _{v \rightarrow v_{0}} \phi\left(v, v_{0}\right)=-\infty
$$

where subindex $v$ indicates the partial derivative with respect to $v$.

Proof. Since the first part is a direct consequence of Equality (2.5), we only show (3.3). Note that $\ell\left(v, v_{0}\right)\left(0 \leq v-v_{0} \ll 1\right)$ is the inverse function of 
$v=v\left(\ell, v_{0}\right)(0 \leq \ell \ll 1)$ which is smooth in $\left(\ell, v_{0}\right)$, which solves

$$
\left(\frac{\ell v_{\ell}}{\sqrt{1+v_{\ell}^{2}}}\right)_{\ell}=-\ell v
$$

with

$$
\lim _{\ell \rightarrow 0} v\left(\ell, v_{0}\right)=v_{0} \quad \text { and } \quad \lim _{\ell \rightarrow 0} v_{\ell}\left(\ell, v_{0}\right)=0
$$

Thus

$$
v\left(\ell\left(v, v_{0}\right), v_{0}\right)=v
$$

holds on the interval $\left(v_{0}, v_{0}+\delta\right)$ with some $\delta>0$. Differentiating (3.4) by $v_{0}$, we obtain

$$
\ell_{v_{0}}\left(v, v_{0}\right)=-\frac{v_{v_{0}}\left(\ell\left(v, v_{0}\right), v_{0}\right)}{v_{\ell}\left(\ell\left(v, v_{0}\right), v_{0}\right)}
$$

for $v \in\left(v_{0}, v_{0}+\delta\right)$. Noting

$$
\ell\left(v, v_{0}\right) \rightarrow 0 \quad \text { as } \quad v \rightarrow v_{0}
$$

and

$$
v_{v_{0}}\left(\ell, v_{0}\right) \rightarrow 1, \quad v_{\ell}\left(\ell, v_{0}\right) \searrow 0 \quad \text { as } \ell \rightarrow 0,
$$

we obtain

$$
\phi\left(v, v_{0}\right)=\ell_{v_{0}}\left(v, v_{0}\right) \rightarrow-\infty \quad \text { as } \quad v \rightarrow v_{0}
$$

Owing to Theorem 4.11 of $[7]$, we have the asymptotic expansion

$$
v(\ell) \sim-\frac{1}{\ell}+\frac{5}{2} \ell^{3}-\frac{567}{8} \ell^{7}+\cdots \quad \text { as } \ell \downarrow 0
$$

for the inverse function of the singular solution $\ell_{\infty}(v)$. Thus we have

$$
\ell_{\infty}(v)=-\frac{1}{v}+O\left(\frac{1}{v^{5}}\right) \quad \text { as } v \rightarrow-\infty
$$

In fact, from (3.5),

$$
v(\ell)=-\frac{1}{\ell}+g(\ell), \quad g(\ell)=O\left(\ell^{3}\right) \quad \text { as } \ell \searrow 0 .
$$


Noting $v \rightarrow-\infty$ if and only if $\ell \searrow 0$, we have

$$
\begin{aligned}
\ell & =-\frac{1}{v-g(\ell)}=-\frac{1}{v}\left[1+\frac{g(\ell)}{v}+O\left(\frac{g(\ell)^{2}}{v^{2}}\right)\right] \\
& =-\frac{1}{v}\left[1+O\left(\frac{1}{v}\right)\right] \quad \text { as } v \rightarrow-\infty .
\end{aligned}
$$

Thus

$$
g(\ell)=O\left(\ell^{3}\right)=O\left(\frac{1}{v^{3}}\right)
$$

and

$$
\ell=-\frac{1}{v}\left[1+\frac{1}{v} O\left(\frac{1}{v^{3}}\right)\right]=-\frac{1}{v}+O\left(\frac{1}{v^{5}}\right) .
$$

Further, noting (3.5), (3.6) and (2.5), we have

$$
\frac{d \ell_{\infty}}{d v}(v)=\frac{1}{v^{2}}+O\left(\frac{1}{v^{6}}\right)
$$

and

$$
\frac{d^{j} \ell_{\infty}}{d v^{j}}(v) \rightarrow 0, \quad(0 \leq j \leq 3) \quad \text { as } \quad v \rightarrow-\infty
$$

These are obtained by carrying out a tedious calculation in practice.

For the limit equation of the variational equation (3.2), we have the following oscillation theorem.

Lemma 3.2. Let $\psi$ be a solution of the equation

$$
\left[\frac{\ell_{\infty}}{\left(1+\ell_{\infty}^{\prime 2}\right)^{3 / 2}} \psi_{v}\right]_{v}+\left(\frac{\ell_{\infty}^{\prime}}{\sqrt{1+\ell_{\infty}^{\prime 2}}}\right)_{v} \psi=v \psi,
$$

where $\ell_{\infty}^{\prime}=\frac{d \ell_{\infty}}{d v}$. Then $\psi$ has infinitely many zeros on the interval $(-\infty, 0)$, that is, (3.9) is oscillatory.

Proof. Putting

$$
a(v)=\frac{\ell_{\infty}(v)}{\left[1+\ell_{\infty}^{\prime 2}\right]^{3 / 2}}, \quad b(v)=\left[\frac{\ell_{\infty}^{\prime}(v)}{\sqrt{1+\ell_{\infty}^{\prime}(v)^{2}}}\right]_{v}-v
$$

we consider the equation

$$
\left(a(v) \psi_{v}(v)\right)_{v}+b(v) \psi(v)=0 \quad \text { on }(-\infty, 0] .
$$


By the Liouville transformation

$$
z(v)=\int_{0}^{v} \frac{d w}{\sqrt{a(w)}}, \quad \Psi(z(v))=a(v)^{1 / 4} \psi(v)
$$

Equation (3.10) turns to

$$
\Psi_{z z}+p(z) \Psi=0 \quad \text { on }(-\xi, 0]
$$

where

$$
p(z(v))=b(v)-\frac{1}{4} a^{\prime \prime}(v)+\frac{1}{16} a(v)^{-1} a^{\prime}(v)^{2}
$$

and

$$
\xi=\int_{-\infty}^{0} \frac{d v}{\sqrt{a(v)}}
$$

Noting (3.6), we have $\xi=\infty$. Thus $z$ tends to $-\infty$ if and only if $v$ goes to $-\infty$. Hence Equation (3.10) is oscillatory at $-\infty$, i.e., any solution has infinitely many zeros on the interval $(-R,-\infty)$ for any $R>0$, if and only if Equation (3.11) is oscillatory. The oscillatory theorem based on the Euler equation (see e.g. [12, Theorem 7.1]) says that if the inequality

$$
\liminf _{z \rightarrow-\infty} z^{2} p(z)>\frac{1}{4}
$$

holds, then Equation (3.11) is oscillatory. In our case, since

$$
p(z(v))=-v+o(1) \quad \text { as } \quad v \rightarrow-\infty
$$

from (3.6), (3.7), (3.8) and $z(v) \rightarrow-\infty$ as $v \rightarrow-\infty$, we have

$$
\lim _{z \rightarrow-\infty} z^{2} p(z)=\lim _{v \rightarrow-\infty} p(z(v))\left(\int_{v}^{0} \frac{d v}{\sqrt{a(v)}}\right)^{2}=\infty .
$$

Thus (3.11), and hence (3.10) is oscillatory.

Now, for a continuous function $\phi$ in $v$, we denote by $i_{(a, b)}(\phi)$ the number of zeros of $\phi$ in the interval $(a, b)$ and put $i\left(v_{0}\right)=i_{\left(v_{0}, 0\right)}\left(\phi\left(\cdot, v_{0}\right)\right)$ for the function $\phi\left(v, v_{0}\right)$ defined by (3.1). Then we have

Lemma 3.3. The number $i\left(v_{0}\right)$ tends to $\infty$ as $v_{0} \rightarrow-\infty$.

Proof. For each $v_{0}<0$, let $\zeta\left(v, v_{0}\right)$ be the solution of $(3.2)$ with the initiat data

$$
\zeta\left(0, v_{0}\right)=0, \quad \frac{\partial \zeta}{\partial v}\left(0, v_{0}\right)=1
$$


Note that the coefficients

$$
\frac{\ell}{\left(1+\ell_{v}^{2}\right)^{3 / 2}}>0 \text { and }\left[\frac{\ell_{v}}{\sqrt{1+\ell_{v}^{2}}}\right]_{v}-v
$$

in (3.2) are smooth on $\left[v_{0}+1,0\right]$, where $\ell=\ell\left(v, v_{0}\right)$. From the Sturmian oscillation theorem, the solutions $\phi\left(v, v_{0}\right)$ and $\zeta\left(v, v_{0} \rightarrow \zeta\left(v, v_{0}\right)\right)$ have one zero between any two consecutive zeros of the other function respectively on the interval $\left[v_{0}+1,0\right]$ or these two functions are linearly dependent. Therefore

$$
i_{\left(v_{0}+1,0\right)}\left(\phi\left(\cdot, v_{0}\right)\right)=i_{\left(v_{0}+1,0\right)}\left(\zeta\left(\cdot, v_{0}\right)\right) \quad \text { or } \quad i_{\left(v_{0}+1,0\right)}\left(\zeta\left(\cdot, v_{0}\right)\right)+1 .
$$

On the other hand, owing to the result obtained by Finn [9], we know that $\ell\left(v, v_{0}\right)$ converges uniformly to a singular solution $\ell_{\infty}(v)$ with all derivatives on any compact set in $(-\infty, 0]$. Thus the coefficients in (3.2) and hence $\zeta\left(v, v_{0}\right)$ converge to the ones and $\psi(v)$, respectively, uniformly with its derivatives in any compact set in $(-\infty, 0]$ as $v_{0} \rightarrow-\infty$. Here $\psi(v)$ is the solution of (3.9) with the initial data

$$
\psi(0)=0, \quad \frac{d \psi}{d v}(0)=1
$$

This implies that, for any fixed $c>0$, there exists a constant $v_{1}(c)<0$ such that

$$
\left|i_{(-c, 0)}\left(\zeta\left(\cdot, v_{0}\right)\right)-i_{(-c, 0)}(\psi)\right| \leq 1
$$

for any $v_{0} \leq v_{1}(c)$. Since $\psi$ has infinitely many zeros on $(-\infty, 0)$ from Lemma 3.2 , there exists a positive constant $c(n)$ for each natural number $n$ such that

$$
i_{(-c(n), 0)}(\psi) \geq n
$$

Thus, taking $c=c(n)$ in (3.15) and $v_{0} \leq \min \left\{-c(n)-1, v_{1}(c(n))\right\}$, we have, from (3.13), (3.14), (3.15),

$$
\begin{aligned}
i\left(v_{0}\right) & \geq i_{(-c(n), 0)}\left(\zeta\left(\cdot, v_{0}\right)\right) \\
& \geq i_{(-c(n), 0)}(\psi)-1 \\
& \geq n-1 .
\end{aligned}
$$

This implies that $i\left(v_{0}\right) \rightarrow \infty$ as $v_{0} \rightarrow-\infty$.

Now we give the proof of Theorem 2.2. 
Proof of Theorem 2.2. From (3.3), we can take a constant $\delta=\delta\left(v_{0}\right)>0$ such that $\phi$ has no zeros on the interval $\left(v_{0}, v_{0}+\delta\right]$. Consider Equation (3.2) on the interval $\left[v_{0}+\delta, 0\right]$. Then the coefficients in (3.2) are continuous and $\ell /\left(1+\ell_{v}^{2}\right)^{3 / 2}$ is strictly positive on $\left[v_{0}+\delta, 0\right]$. Hence the zeros of a solution (3.2) cannot concentrate in the interval $\left[v_{0}+\delta, 0\right]$. Thus any solution of (3.2) has only finite zeros on $\left[v_{0}+\delta, 0\right]$ unless it vanishes identically. This means that $i\left(v_{0}\right)=i_{\left(v_{0}, 0\right)}\left(\phi\left(\cdot, v_{0}\right)\right)$ is finite for each $v_{0}<0$.

We want to show that $\phi\left(0, v_{0}\right)$ changes the sign infinitely many times as $v_{0} \rightarrow-\infty$. Then Theorem 2.2 follows immediately.

For this purpose, we assume that $\phi\left(0, v_{0}\right)$ does not change the sign for $v_{0} \in(a, b)$ with $a<b<0$, but may vanish at some points in $(a, b)$. Then we can show that $i\left(v_{0}\right)$ takes a constant value $i^{*}$ on the interval $(a, b)$ except at the points where $\phi\left(0, v_{0}\right)$ vanishes, and further, the difference between this constant $i^{*}$ and $i\left(v_{0}\right)$ at these zero points of $\phi\left(0, v_{0}\right)$ is at most one. This implies the desired consequence. In fact, $i\left(v_{0}\right)$ tends to $\infty$ as $v_{0} \rightarrow-\infty$ from Lemma 3.3, and the above assertion indicates that there exist infinitely many disjoint intervals $J_{n}=\left[a_{n}, b_{n}\right], 0>b_{1} \geq a_{1}>b_{2} \geq a_{2} \cdots>b_{n} \geq a_{n}>$ $\cdots \rightarrow-\infty$, such that $\phi\left(0, v_{0}\right)=0$ on $J_{n}$ and changes its sign on the both sides of each interval $J_{n}$.

In proving the above fact, first we consider the case that $\phi\left(0, v_{0}\right)$ does not vanish on $(a, b)$. Then, for $\bar{v}_{0} \in(a, b)$, we denote by $\left\{v_{j}\right\}_{1 \leq j \leq m}, 0>v_{1}>$ $v_{2}>\cdots>v_{m}$ the zeros of $\phi\left(v, \bar{v}_{0}\right)$ on $\left(\bar{v}_{0}, 0\right)$, where $m=i\left(\bar{v}_{0}\right)$. Here, each zero point of $\phi\left(v, \bar{v}_{0}\right)$ is not degenerate i.e.,

$$
\frac{\partial \phi}{\partial v}\left(v_{j}, \bar{v}_{0}\right) \neq 0, \quad 1 \leq j \leq m,
$$

because $\phi\left(\cdot, \bar{v}_{0}\right)$ solves the linear equation (3.2) under the boundary condition (3.3), where $v_{0}=\bar{v}_{0}$. Hence, for sufficiently small $\varepsilon>0$, there exists a constant $\delta>0$ such that, if $\left|v_{0}-\bar{v}_{0}\right|<\delta$, then there exists exact one zero of $\phi\left(v, v_{0}\right)$ in each $\varepsilon$-neighborhood of $v_{j}, 1 \leq j \leq m$. This means that $i\left(v_{0}\right)$ is continuous on $(a, b)$, and hence it never varies on $(a, b)$.

Next, we consider the case that $\phi\left(0, v_{0}\right)$ vanishes on $[c, d]$ but does not change sign in a neighborhood of $[c, d]$. When $v_{0}$ varies on a compact set in $(-\infty, 0)$, from Lemma 4.17 and 4.18 in [7], we know that $\ell\left(v, v_{0}\right)$ is extended continuously to $v \in\left(v_{0}, \rho\right)$ with some $\rho>0$ as a solution of (2.5) with (2.6) and $\ell\left(v, v_{0}\right)>0$ there. Let us take $\rho>0$ sufficiently small so that $\phi\left(v, v_{0}\right)>0$ (or $\left.<0\right)$ on $(-\rho, \rho) \backslash\{0\}$ for any $v_{0} \in[c, d]$. In each step we take positive constants $\rho$ and $\delta$ smaller, still denoted by $\rho$ and $\delta$, respectively.

Considering Equation (3.2) on the interval $\left(v_{0}, \rho\right)$ instead of $\left(v_{0}, 0\right)$, we obtain

$$
i_{(c, \rho)}(\phi(\cdot, c))=i_{(d, \rho)}(\phi(\cdot, d))
$$


similarly to the former case. Namely, $i_{(v, \rho)}(\phi(\cdot, v))$ is continuous in $v \in[c, d]$. Because $v=0$ is the only zero point of $\phi(v, c)$ and also of $\phi(v, d)$ in the interval $(-\rho, \rho)$, we have

$$
\begin{aligned}
i(c) & =i_{(c, \rho)}(\phi(\cdot, c))-1 \\
& =i_{(d, \rho)}(\phi(\cdot, d))-1 \\
& =i(d) .
\end{aligned}
$$

We consider the case

$$
\begin{aligned}
\phi\left(0, v_{0}\right) & >0 \text { for } c-\delta<v_{0}<c, d<v_{0}<d+\delta \text { with some } \delta>0 \\
\frac{\partial \phi}{\partial v}(0, c) & >0
\end{aligned}
$$

to prove that

$i(c)=i(d)=i\left(v_{0}\right)-1=i\left(\bar{v}_{0}\right)-1 \quad$ for $c-\delta<v_{0}<c$ and $d<\bar{v}_{0}<d+\delta$.

The other cases

$$
\begin{aligned}
\phi\left(0, v_{0}\right) & >(\text { or }<) 0 \text { for } c-\delta<v_{0}<c, d<v_{0}<d+\delta \\
\frac{\partial \phi}{\partial v}(0, c) & <0
\end{aligned}
$$

will be treated similarly.

For this purpose, we consider Equation (3.2) on the interval $\left(v_{0},-\rho\right)$ and $[-\rho, \rho]$ separately. From the continuity of $(\partial \phi / \partial v)\left(0, v_{0}\right)$ in $v_{0}$,

$$
\frac{\partial \phi}{\partial v}\left(0, v_{0}\right)>0 \text { on }[c-\delta, c+\delta] \text { for small } \delta>0 .
$$

Since $\phi\left(0, v_{0}\right)>0$ on $(c-\delta, c)$ and $(d, d+\delta)$, we have $\phi\left(v, v_{0}\right)>0$ for any $\left(v, v_{0}\right)$ with $0<v<\rho$ with small $\rho>0$ and $c-\delta<v_{0}<c+\delta$. Namely $\phi\left(\cdot, v_{0}\right)$ does not vanish on $[0, \rho]$ for any $v_{0} \in(c-\delta, c+\delta)$. On the other hand, by the same argument as above, if $\rho$ and $\delta$ are taken sufficiently small, then $\phi\left(\cdot, v_{0}\right)$ has exactly one zero point in the interval $[-\rho, \rho]$ for any $v_{0} \in(c-\delta, c+\delta)$. Hence the zero point of $\phi\left(v, v_{0}\right)$ belongs to $[-\rho, 0)$ for $c-\delta<v_{0}<c$ and is equal to zero for $c \leq v_{0}<c+\delta$. This shows

$$
i_{[-\rho, 0)}\left(\phi\left(\cdot, v_{0}\right)\right)=1 \quad \text { and } \quad i_{[-\rho, 0)}(\phi(\cdot, c))=0 .
$$

Noting $\phi\left(-\rho, v_{0}\right) \neq 0$ for $v_{0} \in(c-\delta, c+\delta)$, considering Equation (3.2) on the interval $\left(v_{0},-\rho\right)$ and proceeding the same argument as above, we have

$$
i_{\left(v_{0},-\rho\right)}\left(\phi\left(\cdot, v_{0}\right)\right)=i_{(c,-\rho)}(\phi(\cdot, c)) .
$$


Therefore the equality

$$
\begin{aligned}
i\left(v_{0}\right) & =i_{\left(v_{0},-\rho\right)}\left(\phi\left(\cdot, v_{0}\right)\right)+i_{[-\rho, 0)}\left(\phi\left(\cdot, v_{0}\right)\right) \\
& =i_{(c,-\rho)}(\phi(\cdot, c))+1 \\
& =i(c)+1
\end{aligned}
$$

holds for $c-\delta<v_{0}<c$.

Finally, noting $\phi\left(0, v_{0}\right)=0$ on $[c, d]$, we see that $(\partial \phi / \partial v)\left(0, v_{0}\right)$ does not vanish on $[c, d]$. Hence $(\partial \phi / \partial v)(0, d)>0$. Then, we also have

$$
i\left(v_{0}\right)=i(d)+1 \quad \text { for } d<v_{0}<d+\delta
$$

in the same way as above.

Since $i(c)=i(d)$, we have $i\left(v_{0}\right)=i\left(\bar{v}_{0}\right)$ for any $v_{0} \in(c-\delta, c)$ and $\bar{v}_{0} \in$ $(d, d+\delta)$.

Concluding Remarks. So far, we have proven the infinitely many bendings around $\lambda=\lambda_{*}$ of the bifurcation diagram for the pendent liquid drop (1.6) with (1.7) and (1.8). However, the computational calculation (Figure 4.13 in [7]) suggests the existence of infinitely many solutions at $\lambda=\lambda_{*}$. We have not given a proof for this cojecture yet.

Acknowledgement. The authors wish to thank the referee for valuable advice to improve the manuscript.

\section{References}

[1] F.V. Atkinson and L.A. Peletier, Bounds for vertical points of prescribed mean curvature type equations I, Proc. Roy. Soc. Edinburgh, 112(A) (1989), 15-32.

[2] F.V. Atkinson, L.A. Peletier and J. Serrin, Ground states for prescribed mean curvature equation: the super critical case, In Nonlinear Diffusion Equations and their Equilibrium States, eds. W.-M. Ni, L.A. Peletier and J. Serrin, MSRI Conference Proceedings, Springer-Verlag, New York, 1988.

[3] - Estimates for vertical points of solutions of prescribed mean curvature type equations II, Asymptotic Analysis, 5 (1992), 283-310.

[4] M.F. Bidaut-Veron, Global existence and uniqueness results for singular solutions of the capillarity equation, Pacific J. Math., 125 (1986), 317-333.

[5] P. Concus and R. Finn, Asingular solution of the capillarity equation I. Existence, II. Uniqueness, Invent. Math., 29 (1975), 143-148, 149-159.

[6] The shape of a pendent liquid drop, Philos. Trans. Roy. Soc. London, 292(A) (1979), 307-340.

[7] R. Finn, Equilibrium Capillary Surfaces, Springer-Verlag, New York, 1986. 
[8] - Green identities and pendent liquid drops I, In New Developments in Partial Differential Equations and Applications to Mathematical Physics, Plenum Press, Ferrara, 1991.

[9] _ Green identities and pendent liquid drops II, Proc. Symp. Cont. Mech. and Related Probs, Tbilisi, 1991.

[10] B. Franchi and E. Lanconelli, Radially symmetry of the ground states for a class of quasilinear elliptic equations, In Nonlinear Diffusion Equations and their Equilibrium States, eds. W.-M. Ni, L.A. Peletier and J. Serrin, MSRI Conference Proceedings, Springer-Verlag, New York, 1988.

[11] B. Franchi, E. Lanconelli and J. Serrin, Existence and uniqueness of ground state solutions of quasilinear elliptic equations, In Nonlinear Diffusion Equations and their Equilibrium States, eds. W.-M. Ni, L.A. Peletier and J. Serrin, MSRI Conference Proceedings, Springer-Verlag, New York, 1988.

[12] P. Hartman, Ordinary Differential Equations, 2nd Edition, Birkhäuser, Boston, 1982.

[13] K. Narukawa and T. Suzuki, Nonlinear eigenvalue problem associated to capillary surfaces, Boll. Un. Mat. Ital., 4-B (1990), 223-241.

[14] L.A. Peletier and J. Serrin, Ground states for the prescribed mean curvature equation, Proc. Amer. Math. Soc., 100 (1987), 684-700.

[15] H.C. Wente, The symmetry of sessile and pendent drops, Pacific J. Math., 88 (1980), 387-397.

[16] The stability of the axially symmetric pendent drop, Pacific J. Math., 88 (1980), 421-470.

Received February 25, 1994 and revised May 18, 1994.

NARUTO UNIVERSity OF EdUCATION

NARUTO, TOKUSHIMA, 772

JAPAN

AND

OSAKA UNIVERSITY

TOYONAKA, 560

JAPAN 


\title{
PACIFIC JOURNAL OF MATHEMATICS
}

\author{
Founded in 1951 by
}

\author{
$\begin{array}{ll}\text { E. F. Beckenbach (1906-1982) } & \text { F. Wolf (1904-1989) }\end{array}$
}

\section{EDITORS}

Sun-Yung A. Chang (Managing Editor) Robert Finn University of California

Los Angeles, CA 90095-1555

pacific@math.ucla.edu

\section{F. Michael Christ}

University of California

Los Angeles, CA 90095-1555

christ@math.ucla.edu

Nicholas Ercolani

University of Arizona

Tucson, AZ 85721

ercolani@math.arizona.edu
Stanford University

Stanford, CA 94305

finn@gauss.stanford.edu

Steven Kerckhoff

Stanford University

Stanford, CA 94305

spk@gauss.stanford.edu

Martin Scharlemann

University of California

Santa Barbara, CA 93106

mgscharl@math.ucsb.edu

\section{Gang Tian}

Massachusettes Institute of Technology

Cambridge, MA 02139

tian@math.mit.edu

\section{S. Varadarajan University of California Los Angeles, CA 90095-1555 vsv@math.ucla.edu \\ Dan Voiculescu \\ University of California \\ Berkeley, CA 94720 \\ dvv@math.berkeley.edu}

\section{SUPPORTING INSTITUTIONS}

ACADEMIA SINICA, TAIPEI

CALIF. INST. OF TECHNOLOGY

CHINESE UNIV. OF HONG KONG

HONG KONG UNIV. OF SCI. \& TECH.

KEIO UNIVERSITY

MACQUARIE UNIVERSITY

MATH. SCI. RESEARCH INSTITUTE

NEW MEXICO STATE UNIV.

OREGON STATE UNIV.

PEKING UNIVERSITY

RITSUMEIKAN UNIVERSITY

STANFORD UNIVERSITY

\author{
TOKYO INSTITUTE OF TECHNOLOGY \\ UNIVERSIDAD DE LOS ANDES \\ UNIV. OF ARIZONA \\ UNIV. OF BRITISH COLUMBIA \\ UNIV. OF CALIF., BERKELEY \\ UNIV. OF CALIF., DAVIS \\ UNIV. OF CALIF., IRVINE \\ UNIV. OF CALIF., LOS ANGELES \\ UNIV. OF CALIF., RIVERSIDE \\ UNIV. OF CALIF., SAN DIEGO \\ UNIV. OF CALIF., SANTA BARBARA
}

UNIV. OF CALIF., SANTA CRUZ

UNIV. OF HAWAII

UNIV. OF MELBOURNE

UNIV. OF MONTANA

UNIV. NACIONAL AUTONOMA DE MEXICO

UNIV. OF NEVADA, RENO

UNIV. OF OREGON

UNIV. OF SOUTHERN CALIFORNIA

UNIV. OF UTAH

UNIV. OF WASHINGTON

WASHINGTON STATE UNIVERSITY

The supporting Institutions listed above contribute to the cost of publication of this Journal, but they are not owners or publishers and have no responsibility for its contents or policies.

Manuscripts must be prepared in accordance with the instructions provided on the inside back cover.

The table of contents and the abstracts of the papers in the current issue, as well as other information about the Pacific Journal of Mathematics, may be found on the Internet at http://www.math.uci.edu/pjm.html.

The Pacific Journal of Mathematics (ISSN 0030-8730) is published monthly except for July and August. Regular subscription rate: $\$ 245.00$ a year (10 issues). Special rate: $\$ 123.00$ a year to individual members of supporting institutions.

Subscriptions, back issues published within the last three years and changes of subscribers address should be sent to Pacific Journal of Mathematics, P.O. Box 4163, Berkeley, CA 94704-0163, U.S.A. Prior back issues are obtainable from Kraus Periodicals Co., Route 100, Millwood, NY 10546.

The Pacific Journal of Mathematics at the University of California, c/o Department of Mathematics, 981 Evans Hall, Berkeley, CA 94720 (ISSN 0030-8730) is published monthly except for July and August. Second-class postage paid at Berkeley, CA 94704, and additional mailing offices. POSTMASTER: send address changes to Pacific Journal of Mathematics, P.O. Box 6143, Berkeley, CA 94704-0163.

\section{PUBLISHED BY PACIFIC JOURNAL OF MATHEMATICS at University of California,} Berkeley, CA 94720, A NON-PROFIT CORPORATION

This publication was typeset using AMS-LATEX,

the American Mathematical Society's TEX macro system.

Copyright (C) 1995 by Pacific Journal of Mathematics 


\section{PACIFIC JOURNAL OF MATHEMATICS}

\section{Volume $176 \quad$ No. $2 \quad$ December 1996}

One remark on polynomials in two variables

ENRIQUe ARTAl BARTOLO and PIERRETTE CASSOU-NOGUÈS

Divergence of the normalization for real Lagrangian surfaces near complex tangents XIANGHONG GONG

Classification of the stable homotopy types of stunted lens spaces for an odd prime JESUS GONZALEZ

Plancherel formulae for non-symmetric polar homogeneous spaces

JING-SONG HUANG

A uniqueness theorem for the minimal surface equation

JENN-FANG HWANG

Differential Galois groups of confluent generalized hypergeometric equations: an approach

using Stokes multipliers

Claudine Mitschi

Oscillatory theorem and pendent liquid drops

KIMIAKI NARUKAWA and TAKASHI SUZUKI

Local and global plurisubharmonic defining functions

ALAN NOELL

Specializations and a local homeomorphism theorem for real Riemann surfaces of rings

M. J. DE LA PUENTE

Eigenvalue comparisons in graph theory

GREGORY T. QUENELL

Applications of loop groups and standard modules to Jacobians and theta functions of isospectral curves

WILLI SCHWARZ

Bridged extremal distance and maximal capacity

ROBERT E. THURMAN

Imbedding and multiplier theorems for discrete Littlewood-Paley spaces

IGOR E. VERBITSKY

On constrained extrema

THOMAS VOGEL

Heat flow of equivariant harmonic maps from $\mathbb{B}^{3}$ into $\mathbb{C P} \mathbb{P}^{2}$

YUANLONG XIN

Proof of Longuerre's theorem and its extensions by the method of polar coordinates

ZHIHONG YU

Correction to: "Special generating sets of purely inseparable extension fields of unbounded exponent"

BONIFACE IHEMOTUONYE EKE 